REVISTA INTERNACIONAL DE CIENCIAS DEL DEPORTE International Journal of Sport Science

Rev. int. cienc. deporte

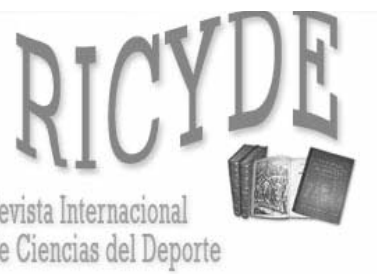

International Journal of Sport Science

VOLUMEN VI - AÑO VI

Páginas:100-111 ISSN:1885-3137

No 19 - Abril - 2010

\title{
Indicadores de rendimiento defensivo en baloncesto en los ganadores y perdedores. Performance indicators defensive basketball winners and losers.
}

\author{
Enrique Ortega Toro \\ Universidad Católica San Antonio de Murcia. España \\ Rodrigo Fernández Verdejo \\ Universidad de Santiago de Chile \\ Marcelo Ubal Rodríguez \\ Universidad Metropolitana de Ciencias de la Educación. Chile \\ Alberto Lorenzo Calvo \\ Universidad Politécnica de Madrid. España \\ Jaime Sampaio \\ Universidad de Trás-os-Montes e Alto Douro. Portugal
}

\section{Resumen}

El objetivo de la presente investigación es analizar las diferencias en la utilización a lo largo del partido de algunos aspectos técnico-tácticos defensivos en baloncesto, entre equipos ganadores y perdedores. Se analizaron todas las fases de ataque $(n=2305)$ llevadas a cabo a lo largo de 12 partidos de la etapa de playoff del campeonato chileno Dimayor jugado el año 2006. En cada fase de ataque se analizaron las siguientes variables en relación con el equipo defensor: (1) Tipo de defensa (individual, zonal, mixta, otras); (2) Presión en la transición ofensiva; (3) Cambios defensivos; (4) Ayudas defensivas; (5) Pases interiores; (6) Grado de oposición al lanzamiento (bajo, medio, alto); (7) Puntos recibidos; (8) Rebotes defensivos; (9) Resultado final del partido. Los resultados más significativos muestran que: a) a lo largo de todo el partido, los equipos ganadores alternan más entre la defensa individual y zonal, mientras que los perdedores alternan entre individual, zonal y mixta; b) las diferencias de puntos en el marcador se producen en el primer y tercer periodo de juego; c) los perdedores presionan más la transición ofensiva en los dos últimos periodos de juego; d) en el cuarto periodo, los equipos perdedores son mejores capturando rebotes defensivos en comparación con los ganadores; e) los cambios defensivos, ayudas sobre el hombre con balón y pases interiores permitidos, no diferencian a ganadores de perdedores en ningún periodo.

Palabras clave: análisis observacional; defensa; rendimiento deportivo.

\section{Abstract}

The object of the present study was to analyze the differences in the use of certain technical and tactical defensive aspects throughout a game in basketball, between winning and losing teams. All attack phases $(n=2305)$ done during 12 play-off games of the 2006 Dimayor Chilean championship were analyzed. In each attack phase the following variables were analyzed in regard to the team in defense: (1) type of defense (man-to-man, zone, combination, other); (2) pressure in the transition offense; (3) defensive switches; (4) defensive help; (5) inside passes; (6) degree of shot opposition (low, medium, high); (7) points received; (8) defensive rebounds; and (9) game result. The most significant results demonstrate that: a) throughout the game, winning teams alternate more between man-to-man and zone defenses, while losing teams alternate between man-to-man, zone, and combination; b) the point differences on the scoreboard occur in the first and third periods of the game; c) losing teams use more pressure in the transition offense in the last two periods of the game; d) in the fourth period, losing teams make more defensive rebounds than winning teams; e) defensive switches, defensive help to guard the player with the ball, and allowing inside passes do not differentiate winning and losing teams in any period of the game.

Key words: observational analysis; defense; athletic performance. 
Ortega, E.; Fernández, R.; Ubal. M.; Lorenzo, A.; Sampaio, J. (2010). Indicadores de rendimiento defensivo en baloncesto en los ganadores y perdedores. Revista Internacional de Ciencias del Deporte. 19(6), $100-111$. http://www.cafyd.com/REVISTA/01901.pdf

\section{Introducción}

$\mathrm{L}^{2}$ a gran mayoría de trabajos de investigación relacionados con el estudio de los diferentes indicadores de rendimiento en el baloncesto, analizan aquellos relacionados con la fase de ataque, apreciándose apenas algunos trabajos en los que se analiza la defensa.

Entre los estudios de acciones defensivas cabe destacar aquellos que analizan los diferentes tipos de sistemas defensivos (individual, zonal, mixta, otros), coincidiendo en la mayoría de los casos que la defensa de tipo individual es la más utilizada (Cruz y Tavares, 1998; Mexas et al., 2005; Farinha y Tavares, 2007). Sólo Silva (1998) encontró que la defensa zonal era más frecuente que la individual (56\% vs $41 \%$ respectivamente) al estudiar jugadores de categoría junior. Diferenciando a ganadores y perdedores, Gómez et al. (2006) hallaron que los primeros utilizaban más la defensa de tipo individual en media cancha, mientras que los segundos se inclinaban más por la presión individual y la defensa mixta. En cuanto a la defensa zonal, estos autores no encontraron diferencias entre los grupos.

Otro de los aspectos más estudiados ha sido la influencia de los rebotes defensivos en el resultado final del juego. La mayoría de los autores han encontrado diferencias significativas a favor de los equipos ganadores en cuanto a la cantidad total de rebotes defensivos capturados (De Rose, 2004; García et al., 2007ª; Ibáñez et al., 2003; Karipidis et al., 2001, Montaner y Montaner, 2004; Sanz y Gutiérrez, 2004). Por otro lado, Forde (2002) encontró una relación significativa entre el éxito en los rebotes y el porcentaje de victorias ( $\mathrm{r}=0.67)$. Gómez et al., (2007) por su parte, al analizar partidos de la Liga ACB y la Liga Femenina, sólo identificó diferencias entre ganadores y perdedores en los playoffs masculinos y la fase regular femenina. Al estudiar tres partidos consecutivos de la liga sub-20 española, García et al. (2007b) encontraron que el rebote defensivo sólo discrimina a ganadores y perdedores en el primer y segundo juego. Sólo Gómez et al. (2006a) encontraron que el rebote no discriminaba entre ganadores y perdedores al analizar partidos de la Liga Femenina Española.

Por otro lado, otra variable defensiva analizada ha sido el grado de oposición al lanzamiento. En este sentido, Ortega et al. (2007) analizaron lanzamientos de tres puntos observando que la oposición parcial era la más frecuente $(59.6 \%)$, por encima de las oposiciones totales $(14.3 \%)$ y los lanzamientos sin oposición (26\%). De igual modo, observaron que los equipos ganadores realizaban mayor porcentaje de lanzamiento de 3 puntos sin oposición que los perdedores, mientras que los equipos perdedores realizaban mayor porcentaje de lanzamientos con oposición máxima que los ganadores. Ortega y Fernández (2007) coinciden con esos resultados, de modo que en su estudio, la oposición parcial fue la más frecuente $(50.5 \%)$, seguida por los lanzamientos sin oposición (38.9\%) y finalmente por la oposición total (10.6\%). En otro estudio, Ibáñez et al. (2007a) muestran que la oposición submáxima fue la más frecuente $(23.9 \%)$ seguida de cerca por oposiciones que llamaron media y elevada (21.9\% y $21.7 \%$, respectivamente).

En cuanto a los pases interiores, sólo se encontró un estudio que analizara su utilización, en el cual se señala que la media de pases interiores por fase de ataque es de solo 0.41 (Cárdenas et al., 1999). Además, en ese mismo estudio no se encontraron diferencias en la utilización del juego interior entre ganadores y perdedores.

A pesar de haber algunas investigaciones que analicen la utilización de los factores defensivos antes mencionados, no todas diferenciaron entre equipos ganadores y perdedores, ni tampoco 
Ortega, E.; Fernández, R.; Ubal. M.; Lorenzo, A.; Sampaio, J. (2010). Indicadores de rendimiento defensivo en baloncesto en los ganadores y perdedores. Revista Internacional de Ciencias del Deporte. 19(6), 100-111. http://www.cafyd.com/REVISTA/01901.pdf

hicieron distinciones entre los diferentes periodos de juego. Debido a que las diferentes situaciones que se dan durante un partido determinan la utilización de los factores defensivos, es muy probable que estos vayan sufriendo variaciones a medida que se acercan los minutos finales en los que se define el resultado. Por lo tanto, realizar un análisis en el que se diferencie la utilización de estos factores según el resultado final y el periodo de juego, permitirá identificar aquellas acciones que son más determinantes en el resultado final en una determinada liga y el momento preciso en que se manifiestan, por lo que sería un dato fundamental para los entrenadores. Además, en relación con otras variables defensivas, como son: la presión en transición ofensiva, ayudas y cambios defensivos, no se encontraron investigaciones previas.

En ese sentido, el objetivo de esta investigación es analizar las diferencias en la utilización a lo largo del partido de algunos factores defensivos entre ganadores y perdedores, con la finalidad de identificar aquellos que son más determinantes del éxito en el baloncesto chileno.

\section{Método}

A través de la metodología observacional (Anguera, 1999, 2004), se analizaron todas las fases de ataque ( $\mathrm{n}=2305)$ llevadas a cabo a lo largo de 12 partidos de la etapa de playoff del campeonato chileno Dimayor jugado el año 2006. Por fase de ataque se consideró aquella que comienza cuando un equipo obtiene la posesión del balón y acaba cuando finalice la posesión del mismo según lo descrito en el Artículo 23 del Reglamento Oficial del Baloncesto (International Basketball Federation, 2000), exceptuando cuando tras un lanzamiento se obtenga un rebote de ataque y se ejecute un lanzamiento casi inmediatamente. Sin embargo, en el caso de existir una falta defensiva que lleve a lanzamientos de tiros libres, estos se consideraron dentro de la misma fase de ataque (Ortega, 2006). Se analizaron a un total de 8 equipos, de manera que del total de fases de ataque, del equipo que más se analizaron, se observaron el $13.5 \%$, mientras que del equipo que se observaron menor número de fases de ataque se registró el 7.1\% del total.

En cada fase de ataque se analizaron las siguientes variables en relación con el equipo defensor: (1) Resultado final (ganador, perdedor); (2) Tipo de defensa (individual, zonal, mixta, otras); (3) Presión en la transición ofensiva, entendido como aquella acción en la que el jugador que transporta el balón de pista trasera a delantera es presionado de manera intensa por su oponente directo; (4) Cambios defensivos, siendo considerados como tal cuando dos defensores intercambian sus atacantes y se mantienen con sus nuevos rivales (sólo en defensas individuales). Se distinguieron 2 tipos de cambios defensivos: puesto-puesto (entre defensores de similares características) e interno-externo (entre defensores de distintas características); (5) Ayudas defensivas, siendo consideradas como tal cuando un defensor que tenía asignado otro atacante deja a éste de lado y se dirige a enfrentar al hombre con balón que ha sacado cierta ventaja sobre su marcador directo (sólo en defensas individuales); (6) Pases interiores, considerados como aquellos pases que se reciben dentro de las zonas cercana, próxima o próxima trasera (según las divisiones del campo realizadas por Ortega, 2006); (7) Grado de oposición al lanzamiento (bajo, medio, alto); (8) Puntos recibidos; (9) Rebotes defensivos.

Para la recogida de los datos se utilizó, siguiendo la propuesta de Anguera $(1999,2004)$, un sistema de categorías. Posteriormente se realizó un entrenamiento de los observadores, para lo cual se siguieron los criterios descritos por Behar (1993). Los observadores obtuvieron una 
Ortega, E.; Fernández, R.; Ubal. M.; Lorenzo, A.; Sampaio, J. (2010). Indicadores de rendimiento defensivo en baloncesto en los ganadores y perdedores. Revista Internacional de Ciencias del Deporte. 19(6), $100-111$. http://www.cafyd.com/REVISTA/01901.pdf

fiabilidad mínima del 0.99 a nivel inter e intra-observador (índice de Kappa y/o coeficiente de correlación intraclase) al final del proceso de entrenamiento. El análisis de los datos se realizó con el programa SPSS 15 para Windows, utilizándose la prueba de Chi-cuadrado para buscar relaciones entre ganadores y perdedores. De igual modo, se usó la prueba U de Mann-Whitney para buscar diferencias entre ganadores y perdedores en las variables con nivel de medición de razón (numéricas) en cada periodo de juego. En todos los casos se utilizó una significación de $\mathrm{p}<0.05$.

\section{Resultados}

Las frecuencias de utilización por periodo para ganadores y perdedores de las variables tipo de defensa, presión en transición, grado de oposición y rebote defensivo se presentan en la tabla 1.

\begin{tabular}{|c|c|c|c|c|c|c|c|c|c|c|c|}
\hline \multirow{2}{*}{ Variables } & \multirow{2}{*}{ Categorías } & \multicolumn{2}{|c|}{$1^{\circ}$ Periodo } & \multicolumn{2}{|c|}{$2^{\circ}$ Periodo } & \multicolumn{2}{|c|}{$3^{\circ}$ Periodo } & \multicolumn{2}{|c|}{$4^{\circ}$ Periodo } & \multicolumn{2}{|c|}{ Total } \\
\hline & & Gana & Pierde & Gana & Pierde & Gana & Pierde & Gana & Pierde & Gana & Pierde \\
\hline \multirow{5}{*}{$\begin{array}{l}\text { Tipo de } \\
\text { defensa }^{A}\end{array}$} & Individual & $\begin{array}{c}172 \\
(59.7 \%) \\
\end{array}$ & $\begin{array}{c}181 \\
(63.3 \%)\end{array}$ & $\begin{array}{c}134 \\
(48.2 \%)\end{array}$ & $\begin{array}{c}147 \\
(51.4 \%) \\
\end{array}$ & $\begin{array}{c}127 \\
(44.4 \%)\end{array}$ & $\begin{array}{c}177 \\
(62.5 \%)\end{array}$ & $\begin{array}{c}178 \\
(58.4 \%)\end{array}$ & $\begin{array}{c}186 \\
(64.4 \%)\end{array}$ & $\begin{array}{c}675 \\
(53.7 \%)\end{array}$ & $\begin{array}{c}744 \\
(59.4 \%)\end{array}$ \\
\hline & Zonal & $\begin{array}{c}64 \\
(22.2 \%)\end{array}$ & $\begin{array}{c}38 \\
(13.3 \%)\end{array}$ & $\begin{array}{c}85 \\
(30.6 \%)\end{array}$ & $\begin{array}{c}30 \\
(10.5 \%)\end{array}$ & $\begin{array}{c}93 \\
(32.5 \%)\end{array}$ & $\begin{array}{c}12 \\
(4.2 \%)\end{array}$ & $\begin{array}{c}53 \\
(17.4 \%)\end{array}$ & $\begin{array}{c}29 \\
(10.0 \%)\end{array}$ & $\begin{array}{c}308 \\
(24.5 \%)\end{array}$ & $\begin{array}{c}136 \\
(10.9 \%)\end{array}$ \\
\hline & Mixta & $\begin{array}{c}0 \\
(0 \%)\end{array}$ & $\begin{array}{c}0 \\
(0 \%)\end{array}$ & $\begin{array}{c}0 \\
(0 \%)\end{array}$ & $\begin{array}{c}53 \\
(18.5 \%)\end{array}$ & $\begin{array}{c}13 \\
(4.5 \%)\end{array}$ & $\begin{array}{c}32 \\
(11.3 \%)\end{array}$ & $\begin{array}{c}3 \\
(1.0 \%)\end{array}$ & $\begin{array}{c}11 \\
(3.8 \%)\end{array}$ & $\begin{array}{c}16 \\
(1.3 \%)\end{array}$ & $\begin{array}{c}96 \\
(7.7 \%)\end{array}$ \\
\hline & Otras & $\begin{array}{c}52 \\
(18.1 \%) \\
\end{array}$ & $\begin{array}{c}67 \\
(23.4 \%) \\
\end{array}$ & $\begin{array}{c}59 \\
(21.2 \%) \\
\end{array}$ & $\begin{array}{c}56 \\
(19.6 \%)\end{array}$ & $\begin{array}{c}53 \\
(18.5 \%) \\
\end{array}$ & $\begin{array}{c}62 \\
(21.9 \%)\end{array}$ & $\begin{array}{c}71 \\
(23.3 \%)\end{array}$ & $\begin{array}{c}63 \\
(21.8 \%)\end{array}$ & $\begin{array}{c}258 \\
(20.5 \%)\end{array}$ & $\begin{array}{c}276 \\
(22.0 \%)\end{array}$ \\
\hline & $\mathrm{N}$ & 288 & 286 & 278 & 286 & 286 & 283 & 305 & 289 & 1257 & 1252 \\
\hline \multirow{3}{*}{$\begin{array}{l}\text { Presión en } \\
\text { transición } \\
\text { ofensiva }^{B}\end{array}$} & $\mathrm{Si}$ & $\begin{array}{c}31 \\
(13 \%)\end{array}$ & $\begin{array}{c}27 \\
(11.4 \%)\end{array}$ & $\begin{array}{c}42 \\
(17.5 \%)\end{array}$ & $\begin{array}{c}38 \\
(16.1 \%)\end{array}$ & $\begin{array}{c}34 \\
(15.2 \%) \\
\end{array}$ & $\begin{array}{c}55 \\
(24.4 \%) \\
\end{array}$ & $\begin{array}{c}28 \\
(12.4 \%)\end{array}$ & $\begin{array}{c}86 \\
(37.6 \%) \\
\end{array}$ & $\begin{array}{c}146 \\
(14.5 \%)\end{array}$ & $\begin{array}{c}216 \\
(21.4 \%)\end{array}$ \\
\hline & No & $\begin{array}{c}207 \\
(87 \%)\end{array}$ & $\begin{array}{c}210 \\
(88.6 \%)\end{array}$ & $\begin{array}{c}198 \\
(82.5 \%)\end{array}$ & $\begin{array}{c}198 \\
(83.9 \%)\end{array}$ & $\begin{array}{c}190 \\
(84.8 \%)\end{array}$ & $\begin{array}{c}170 \\
(75.6 \%)\end{array}$ & $\begin{array}{c}198 \\
(87.6 \%)\end{array}$ & $\begin{array}{c}143 \\
(62.4 \%)\end{array}$ & $\begin{array}{c}859 \\
(85.5 \%)\end{array}$ & $\begin{array}{c}791 \\
(78.6 \%)\end{array}$ \\
\hline & $\mathrm{N}$ & 238 & 237 & 240 & 236 & 224 & 225 & 226 & 229 & 1005 & 1007 \\
\hline \multirow{4}{*}{$\begin{array}{l}\text { Grado de } \\
\text { oposición al } \\
\text { lanzamiento }{ }^{C}\end{array}$} & Bajo & $\begin{array}{c}104 \\
(59.1 \%)\end{array}$ & $\begin{array}{c}107 \\
(64.8 \%)\end{array}$ & $\begin{array}{c}88 \\
(59.5 \%)\end{array}$ & $\begin{array}{c}102 \\
(60.7 \%)\end{array}$ & $\begin{array}{c}106 \\
(60.2 \%)\end{array}$ & $\begin{array}{c}104 \\
(63.0 \%)\end{array}$ & $\begin{array}{c}110 \\
(61.1 \%)\end{array}$ & $\begin{array}{c}99 \\
(66.9 \%)\end{array}$ & $\begin{array}{c}437 \\
(59.8 \%)\end{array}$ & $\begin{array}{c}455 \\
(63.1 \%)\end{array}$ \\
\hline & Medio & $\begin{array}{c}38 \\
(21.6 \%)\end{array}$ & $\begin{array}{c}17 \\
(10.3 \%)\end{array}$ & $\begin{array}{c}29 \\
(19.6 \%)\end{array}$ & $\begin{array}{c}27 \\
(16.1 \%)\end{array}$ & $\begin{array}{c}27 \\
(15.3 \%)\end{array}$ & $\begin{array}{c}22 \\
(13.3 \%)\end{array}$ & $\begin{array}{c}24 \\
(13.3 \%)\end{array}$ & $\begin{array}{c}13 \\
(8.8 \%)\end{array}$ & $\begin{array}{c}130 \\
(17.8 \%)\end{array}$ & $\begin{array}{c}90 \\
(12.5 \%)\end{array}$ \\
\hline & Alto & $\begin{array}{c}34 \\
(19.3 \%)\end{array}$ & $\begin{array}{c}41 \\
(24.8 \%)\end{array}$ & $\begin{array}{c}31 \\
(20.9 \%)\end{array}$ & $\begin{array}{c}39 \\
(23.2 \%) \\
\end{array}$ & $\begin{array}{c}43 \\
(24.4 \%)\end{array}$ & $\begin{array}{c}39 \\
(23.6 \%)\end{array}$ & $\begin{array}{c}46 \\
(25.6 \%)\end{array}$ & $\begin{array}{c}36 \\
(24.3 \%)\end{array}$ & $\begin{array}{c}164 \\
(22.4 \%)\end{array}$ & $\begin{array}{c}176 \\
(24.4 \%)\end{array}$ \\
\hline & $\mathrm{N}$ & 176 & 165 & 148 & 168 & 176 & 165 & 180 & 148 & 731 & 721 \\
\hline \multirow{3}{*}{$\begin{array}{l}\text { Rebotes } \\
\text { defensivos }\end{array}$} & $\mathrm{Si}$ & $\begin{array}{c}76 \\
(74.5 \%) \\
\end{array}$ & $\begin{array}{c}46 \\
(67.7 \%) \\
\end{array}$ & $\begin{array}{c}60 \\
(74.1 \%) \\
\end{array}$ & $\begin{array}{c}67 \\
(71.3 \%) \\
\end{array}$ & $\begin{array}{c}74 \\
(72.5 \%)\end{array}$ & $\begin{array}{c}50 \\
(61.7 \%) \\
\end{array}$ & $\begin{array}{c}57 \\
(55.9 \%) \\
\end{array}$ & $\begin{array}{c}57 \\
(75 \%)\end{array}$ & $\begin{array}{c}286 \\
(68.8 \%)\end{array}$ & $\begin{array}{c}240 \\
(67.2 \%)\end{array}$ \\
\hline & No & $\begin{array}{c}26 \\
(25.5 \%)\end{array}$ & $\begin{array}{c}22 \\
(32.4 \%)\end{array}$ & $\begin{array}{c}21 \\
(25.9 \%)\end{array}$ & $\begin{array}{c}27 \\
(28.7 \%)\end{array}$ & $\begin{array}{c}28 \\
(27.5 \%)\end{array}$ & $\begin{array}{c}31 \\
(38.3 \%)\end{array}$ & $\begin{array}{c}45 \\
(44.1 \%)\end{array}$ & $\begin{array}{c}19 \\
(25 \%)\end{array}$ & $\begin{array}{c}130 \\
(31.2 \%)\end{array}$ & $\begin{array}{c}117 \\
(32.8 \%)\end{array}$ \\
\hline & $\mathrm{N}$ & 102 & 68 & 81 & 94 & 102 & 81 & 102 & 76 & 416 & 357 \\
\hline
\end{tabular}

En la tabla 1 se puede apreciar que la defensa de tipo individual es la más utilizada tanto por ganadores como por perdedores con independencia del periodo de juego. Además, se encontró relación entre el resultado final y tipo de defensa a lo largo de todos los periodos de juego, de modo que en el primer periodo los equipos ganadores realizan mayor cantidad de defensas zonales, pero menos individuales y otras en comparación con los perdedores $\left(\chi^{2}(2)=8.741\right.$, $\mathrm{p}<0.02)$. En el segundo periodo, los ganadores se mantienen realizando más defensas zonales y menos individuales, además de menos defensas mixtas, pero esta vez superan a los perdedores en 
Ortega, E.; Fernández, R.; Ubal. M.; Lorenzo, A.; Sampaio, J. (2010). Indicadores de rendimiento defensivo en baloncesto en los ganadores y perdedores. Revista Internacional de Ciencias del Deporte. 19(6), $100-111$. http://www.cafyd.com/REVISTA/01901.pdf

la cantidad de utilización de otras defensas $\left(\chi^{2}(3)=79.887, \mathrm{p}<0.01\right)$. En el tercer periodo los equipos ganadores sólo superan a los perdedores en la frecuencia de las defensas zonales, obteniendo valores inferiores en los otros tres tipos de defensas $\left(\chi^{2}(3)=79.442, p<0.01\right)$. Por último, en el cuarto periodo se repite la situación del segundo periodo, en la que los ganadores son superiores a los perdedores en las defensas de tipo zonal y otras $\left(\chi^{2}(3)=11.827, p<0.01\right)$. Por lo tanto, se observa que los equipos ganadores, a lo largo de todo el juego, realizan menor cantidad de defensas de tipo individual y mixta, pero una mayor cantidad de defensas zonales en comparación con los perdedores.

En cuanto a la presión en transición, los datos señalan que es muy poco utilizada, presionándose menos del $40 \%$ de las transiciones independientemente del resultado final o del periodo de juego (tabla 1). Se encontraron relaciones significativas entre ganadores y perdedores sólo en la segunda mitad del partido, de modo que los perdedores presionan más la transición en comparación con los ganadores en el tercer $\left(\chi^{2}(1)=6.064, p<0.02\right) \quad$ y cuarto periodos $\left(\chi^{2}(1)=38.362, p<0.01\right)$.

La tabla 1 muestra que el grado de oposición bajo es el más frecuente independientemente del resultado final o el periodo de juego. Se encontró relación entre la oposición al lanzamiento y el resultado final sólo en el primer periodo, de manera que los equipos ganadores realizan una mayor cantidad de oposiciones medias, pero menos oposiciones altas y bajas en comparación con los perdedores $\left(\chi^{2}(2)=8.386, \mathrm{p}<0.02\right)$.

En cuanto al rebote defensivo, la tabla 1 señala que se capturan en más del $60 \%$ de las oportunidades que se tienen para hacerlo, independientemente del resultado final o el periodo de juego. Sólo se encontró relación entre el resultado final y el periodo de juego en el cuarto periodo, indicando que en ese momento los perdedores son mejores capturando rebotes defensivos $\left(\chi^{2}(1)=6.912, p<0.01\right)$.

La tabla 2 muestra los resultados por periodo de ganadores y perdedores en las variables cambios defensivos, ayudas, pases interiores permitidos y puntos recibidos.

\begin{tabular}{|c|c|c|c|c|c|c|c|c|c|c|c|}
\hline \multirow{2}{*}{ Variables } & \multirow{2}{*}{ Categorías } & \multicolumn{2}{|c|}{$1^{\circ}$ Periodo } & \multicolumn{2}{|c|}{$2^{\circ}$ Periodo } & \multicolumn{2}{|c|}{$3^{\circ}$ Periodo } & \multicolumn{2}{|c|}{$4^{\circ}$ Periodo } & \multicolumn{2}{|c|}{ Total } \\
\hline & & Gana & Pierde & Gana & Pierde & Gana & Pierde & Gana & Pierde & Gana & Pierde \\
\hline \multirow{4}{*}{$\begin{array}{l}\text { Cambios } \\
\text { defensivos }\end{array}$} & $\begin{array}{l}\text { Puesto- } \\
\text { puesto }\end{array}$ & $0.06 \pm 0.2$ & $0.05 \pm 0.2$ & $0.02 \pm 0.1$ & $0.05 \pm 0.2$ & $0.07 \pm 0.2$ & $0.07 \pm 0.2$ & $0.07 \pm 0.3$ & $0.11 \pm 0.3$ & $0.06 \pm 0.2$ & $0.07 \pm 0.2$ \\
\hline & $\begin{array}{l}\text { Interno- } \\
\text { externo }\end{array}$ & $0.06 \pm 0.3$ & $0.06 \pm 0.2$ & $0.05 \pm 0.2$ & $0.05 \pm 0.2$ & $0.09 \pm 0.3$ & $0.09 \pm 0.3$ & $0.09 \pm 0.3$ & $0.10 \pm 0.3$ & $0.08 \pm 0.3$ & $0.08 \pm 0.2$ \\
\hline & Totales & $0.12 \pm 0.4$ & $0.11 \pm 0.3$ & $0.08 \pm 0.2$ & $0.10 \pm 0.3$ & $0.16 \pm 0.4$ & $0.15 \pm 0.4$ & $0.15 \pm 0.4$ & $0.21 \pm 0.4$ & $0.14 \pm 0.4$ & $0.15 \pm 0.3$ \\
\hline & $\mathrm{N}$ & 170 & 180 & 133 & 146 & 126 & 175 & 176 & 185 & 669 & 739 \\
\hline \multirow{2}{*}{$\begin{array}{l}\text { Ayudas } \\
\text { defensivas }\end{array}$} & & $0.52 \pm 0.8$ & $0.49 \pm 0.7$ & $0.48 \pm 0.7$ & $0.47 \pm 0.7$ & $0.52 \pm 0.7$ & $0.54 \pm 0.8$ & $0.61 \pm 0.8$ & $0.59 \pm 0.8$ & $0.54 \pm 0.8$ & $0.51 \pm 0.7$ \\
\hline & $\mathrm{N}$ & 171 & 178 & 134 & 146 & 125 & 175 & 174 & 186 & 667 & 738 \\
\hline \multirow{2}{*}{$\begin{array}{l}\text { Pases } \\
\text { interiores } \\
\text { permitidos }\end{array}$} & & $0.13 \pm 0.3$ & $0.15 \pm 0.3$ & $0.17 \pm 0.3$ & $0.15 \pm 0.3$ & $0.19 \pm 0.4$ & $0.20 \pm 0.4$ & $0.15 \pm 0.3$ & $0.17 \pm 0.3$ & $0.16 \pm 0.4$ & $0.18 \pm 0.3$ \\
\hline & $\mathrm{N}$ & 287 & 286 & 278 & 285 & 286 & 281 & 305 & 288 & 1256 & 1248 \\
\hline \multirow{2}{*}{$\begin{array}{l}\text { Puntos } \\
\text { recibidos* }\end{array}$} & & $0.75 \pm 1.0$ & $1.03 \pm 1.1^{*}$ & $0.80 \pm 1.0$ & $0.77 \pm 1.0$ & $0.78 \pm 1.0$ & $0.99 \pm 1.1^{*}$ & $0.87 \pm 1.1$ & $0.84 \pm 1.0$ & $0.79 \pm 1.0$ & $0.91 \pm 1.1$ \\
\hline & $\mathrm{N}$ & 288 & 286 & 279 & 287 & 286 & 284 & 306 & 289 & 1259 & 1254 \\
\hline
\end{tabular}


Ortega, E.; Fernández, R.; Ubal. M.; Lorenzo, A.; Sampaio, J. (2010). Indicadores de rendimiento defensivo en baloncesto en los ganadores y perdedores. Revista Internacional de Ciencias del Deporte. 19(6), $100-111$. http://www.cafyd.com/REVISTA/01901.pdf

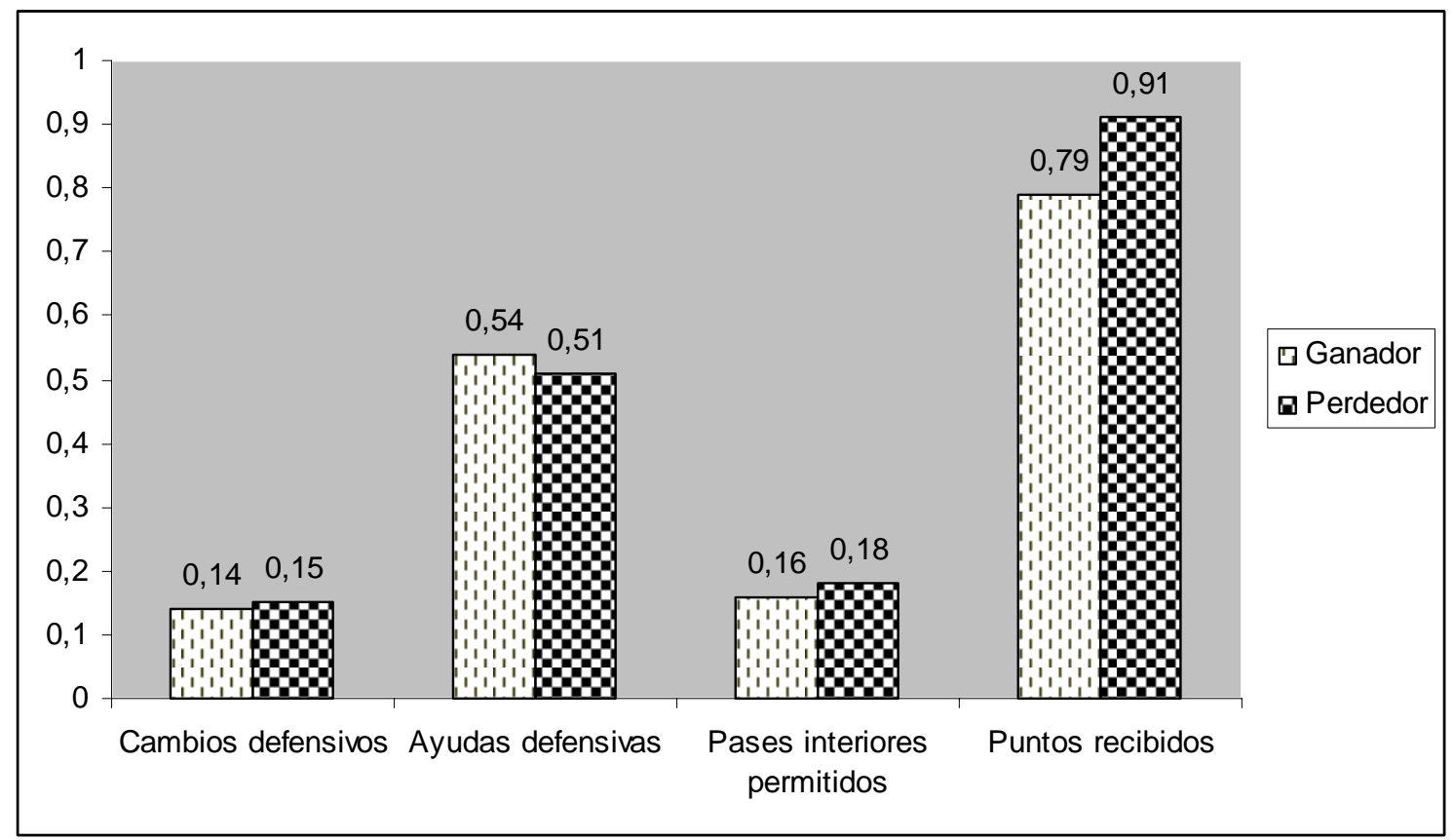

Figura 1.- Valores medios de las variables objeto de estudio en el total del partido.

De la tabla 2 y la figura 1, cabe destacar los escasos valores registrados de las diferentes variables en la muestra objeto de estudio con independencia del resultado final o del periodo de juego. No se encontraron diferencias significativas entre ganadores y perdedores en ninguno de los periodos de juego en las variables: cambios defensivos, ayudas, ni pases interiores.

Hubo diferencias entre ganadores y perdedores en el primer $(\mathrm{Z}=-3.128, \mathrm{p}<0.01)$ y tercer $(\mathrm{Z}=-$ $2.275, \mathrm{p}<0.03$ ) periodo en cuanto al número de puntos recibidos (tabla 2). Por lo tanto, los perdedores reciben más puntos que los ganadores en esos momentos del partido.

\section{Discusión}

Los resultados de esta investigación muestran que las defensas de tipo individual y zonal son las más utilizadas tanto por ganadores como por perdedores, siendo la individual muy superior (más del $50 \%$ de las fases de ataque para ambos grupos). No obstante, se encontraron diferencias significativas en la utilización de las defensas entre los grupos, de manera que los ganadores usan en mayor medida la defensa zonal y muy poco la de tipo mixta; los perdedores en cambio, alternan más entre los tres tipos de defensas. Esta situación se repite a lo largo de los cuatro periodos de juego.

Se observa entonces que los ganadores presentan una tendencia a utilizar en mayor medida la defensa individual, seguida por la zonal, y finalmente la mixta. Las razones de esto pueden estar en que la continua variación entre las defensas individual y zonal dificultaría la organización de los ataques, obligándolos a cambiar sus estrategias constantemente. Que los equipos perdedores alternen más entre las tres defensas puede ser debido a que al quedar debajo en el marcador buscan todos los recursos posibles para remontar. 
Ortega, E.; Fernández, R.; Ubal. M.; Lorenzo, A.; Sampaio, J. (2010). Indicadores de rendimiento defensivo en baloncesto en los ganadores y perdedores. Revista Internacional de Ciencias del Deporte. 19(6), 100-111. http://www.cafyd.com/REVISTA/01901.pdf

Otras investigaciones también encontraron resultados similares en otros contextos, siendo la defensa individual la más utilizada entre los equipos (Cruz y Tavares, 1998; Mexas et al., 2005). Farinha y Tavares (2007) analizaron a ganadores y perdedores del campeonato nacional senior de la liga portuguesa, y al igual que en este estudio, encontraron que en ambos grupos la defensa individual era la más utilizada. Sólo Silva (1998) encontró que la defensa zonal era más usada que la individual, pero la muestra utilizada (jugadores junior) podría explicar en parte esta situación.

De igual modo, Gómez et al. (2006b) sobre una muestra del baloncesto español, también descubrieron que los equipos perdedores realizan más defensas mixtas que los ganadores, sin embargo no encontraron diferencias en la utilización de la defensa zonal.

En cuanto a la presión en transición ofensiva, este factor defensivo fue más utilizado por los equipos perdedores que por los ganadores. Esas diferencias se presentaron en los dos últimos periodos de juego, lo que podría estar indicando que los equipos que están en desventaja en el marcador optan por presionar a sus rivales a medida que el partido está llegando a su fin, para de ese modo intentar disminuir las diferencias. Finalmente son esos los equipos que terminan perdiendo el juego, lo que explica en parte los resultados hallados.

Por lo tanto, los datos no deben interpretarse como que no se debe presionar la transición para ganar un partido, sino que debe entenderse que esa presión es un recurso para remontar un marcador adverso.

Se encontraron diferencias entre ganadores y perdedores sólo en el primer periodo en cuanto a la utilización de los diferentes grados de oposición al lanzamiento. De modo que, si bien el grado de oposición bajo es el más frecuente tanto para ganadores como para perdedores, los primeros son ampliamente superiores en cuanto a la utilización del grado de oposición medio, siendo los segundos superiores en las oposiciones bajas y altas. Lo que indica que los equipos ganadores no son tan extremos en relación con su oposición al lanzamiento, de manera que constantemente estarían intentando molestar a sus rivales aunque eso no signifique llegar a bloquearlos totalmente. En cambio, los equipos perdedores siguen una tendencia más hacia los extremos en la que se esfuerzan más por oponerse a un tiro cuando sienten que tienen la posibilidad real de bloquearlo, si eso no es así simplemente dejan al atacante ejecutar su lanzamiento. Por otro lado, estos datos pueden deberse a que los equipos ganadores consiguen organizar mejor su ataque obteniendo tiros sin oposición.

En este sentido, el que en el primer periodo los equipos perdedores realicen más oposiciones bajas en comparación con los ganadores indica que en realidad se oponen a menos lanzamientos (la categoría oposición baja indica que casi no hubo oposición). Las investigaciones previas muestran que a mayor oposición hay menor eficacia (Ibáñez et al., 2007a, 2007b; Ortega et al., 2007), lo que indica que en el primer periodo de juego es muy probable que los ganadores obtengan mayor eficacia en sus lanzamientos, ya que los perdedores se oponen a menos lanzamientos. Esto último se reafirma al analizar los datos sobre el número de puntos recibidos.

En cuanto a los rebotes defensivos, se debe comentar que la metodología utilizada para el registro y valoración de esta variable fue distinta a la usada en otros estudios. En vez de registrar el valor absoluto de rebotes defensivos en cada partido, se calculó el porcentaje de veces en que se 
Ortega, E.; Fernández, R.; Ubal. M.; Lorenzo, A.; Sampaio, J. (2010). Indicadores de rendimiento defensivo en baloncesto en los ganadores y perdedores. Revista Internacional de Ciencias del Deporte. 19(6), $100-111$. http://www.cafyd.com/REVISTA/01901.pdf

capturó un rebote defensivo en relación con el total de fases en las que se podrían haber capturado (fase que finalizan con lanzamiento o tapón, y además no se anotan puntos).

Los resultados indican que sólo hubo diferencias en cuanto a los rebotes defensivos capturados entre ganadores y perdedores en el último periodo de juego. De modo que en ese momento los perdedores son mejores tomando rebotes en defensa.

Investigaciones previas han mostrado que en determinadas situaciones no hay diferencias entre ganadores y perdedores en los rebotes defensivos. Gómez et al. (2006a) no encontraron diferencias al analizar el baloncesto femenino español. Gómez (2007) encontró sólo diferencias en los partidos de playoff masculinos y de fase regular femenina, pero no en los playoff femeninos ni en la fase regular masculina. García et al. (2007b), al analizar tres partidos en días consecutivos, encontró diferencias en el primer y segundo partido, pero no en el tercero.

En cambio, investigaciones que sí encontraron que los rebotes eran influyentes en el resultado final son bastantes, de manera que los ganadores atrapan más rebotes que los perdedores (De Rose, 2004; Forde, 2002; García et al., 2007a; Ibáñez et al., 2003; Karipidis et al., 2001; Montaner y Montaner, 2004; Sanz y Gutiérrez, 2004).

No obstante en esta investigación los resultados muestran todo lo contrario, en cuanto que son los perdedores mejores capturando rebotes en el cuarto periodo. La explicación para los contradictorios resultados se cree está en la metodología utilizada. Ahora bien, que los perdedores atrapen más rebotes en los momentos finales del juego podría indicar que al final del partido los equipos en desventaja maximizan sus esfuerzos para remontar el marcador.

Producto de las acciones tácticas ofensivas, los defensores pueden verse obligados a cambiar a sus atacantes (en defensa individual). Esta es una situación indeseable para los equipos defensores, ya que supuestamente las marcaciones individuales fueron asignadas de la manera más conveniente. Los resultados de este estudio indican que este factor defensivo es muy poco utilizado, no encontrándose diferencias entre ganadores y perdedores en ninguno de los periodos. Las medias de todos los cambios defensivos (puesto-puesto, interno-externo, totales) a lo largo de los cuatro periodos de juego, tanto para ganadores como perdedores, son inferiores a 1. En ese sentido, se podría especular que las defensas chilenas superan en este aspecto a los ataques, ya que estos últimos no son capaces de forzar cambios defensivos que pudiesen ser beneficiosos para ellos. Sin embargo, también está la posibilidad de que los equipos atacantes no tienen dentro de sus prioridades forzar los cambios defensivos.

Uno de los aspectos que la bibliografía remarca como clave defensiva, es el uso de las ayudas defensivas. En este caso, tampoco hubo diferencias entre ganadores y perdedores en ningún periodo de juego y los valores encontrados son inferiores a 1 en todos los casos. Lamentablemente no se encontraron datos con los que comparar estos resultados, pero tomando en cuenta la condición colectiva de este deporte, la cantidad de ayudas defensivas es muy baja. Una de las posibles razones para esta situación es que los defensores analizados, independientemente del resultado o el periodo, no juegan en equipo, y se preocupan más de que su atacante no anote en vez de velar por evitar los puntos del equipo rival en su conjunto. Otra explicación podría encontrarse en que las situaciones de definición en el baloncesto chileno se dan en enfrentamientos en los que el atacante no toma una real ventaja sobre su defensor y, por lo tanto, no se requiere ayuda (las ayudas sólo fueron registradas si es que el atacante tomaba 
Ortega, E.; Fernández, R.; Ubal. M.; Lorenzo, A.; Sampaio, J. (2010). Indicadores de rendimiento defensivo en baloncesto en los ganadores y perdedores. Revista Internacional de Ciencias del Deporte. 19(6), $100-111$. http://www.cafyd.com/REVISTA/01901.pdf

ventaja de su defensor). Sin embargo, esta última hipótesis parece no ser tan probable al comparar estos datos con los del grado de oposición vistos anteriormente. Al igual que ocurre con los cambios defensivos, a pesar de la tremenda importancia de las ayudas defensivas, no se han encontrado trabajos de investigación que hagan alusión a este importante contenido técnicotáctico colectivo defensivo.

Otro aspecto poco estudiado es el uso de pases interiores. A partir de los resultados obtenidos se puede ver que en el baloncesto chileno no es muy utilizado o permitido el pase interior. En el tercer periodo los perdedores promedian 0.2 pases interiores recibidos, siendo ese el mayor valor obtenido, lo que significa dos pases interiores cada diez fases de ataque. No hubo diferencias entre ganadores y perdedores en ninguno de los periodos de juego, lo que indica que la utilización de este factor no sería tan determinante del resultado final. Similares resultados encontraron Cárdenas et al. (1999), quienes al analizar partidos de la liga ACB tampoco encontraron diferencias en el uso del juego interior entre equipos ganadores y perdedores. Por lo tanto, los pases interiores recibidos no serían un factor determinante del éxito.

En el primer y tercer periodo se presentaron diferencias significativas entre el número de puntos permitidos por fase de ataque entre ganadores y perdedores, de manera que los primeros permiten menos. Que los ganadores reciban menos puntos es obvio considerando que para ganar el partido se requiere anotar más puntos que el rival. Otras investigaciones ya habían encontrado diferencias al analizar datos totales de juegos (Ortega et al., 2006; Gómez et al., 2006b). Sin embargo, lo interesante de los datos obtenidos aquí es que las diferencias se presentaron sólo en el primer y tercer periodo, lo que indica que es en esos momentos cuando los equipos ganadores toman ventajas en el marcador sobre sus rivales.

Así, Ortega et al. (2007), encontraron diferencias a favor de los ganadores en la eficacia del lanzamiento de tres puntos en el primero ( $34.6 \%$ vs $30.9 \%)$, segundo $(33.3 \%$ vs $22.6 \%$ ) y cuarto ( $28.3 \%$ vs $20.0 \%$ ) periodos, pero no en el tercero (30.1\% vs $30.3 \%)$. Esto indica que no se sigue una tendencia, sino que más bien cada liga presenta características propias.

Los datos sobre los puntos recibidos encontrados aquí se pueden relacionar con otras variables de este mismo estudio. Se mencionó que justamente en el primer periodo es cuando los perdedores presionan menos los lanzamientos, lo que facilitaría a los ganadores las labores de anotación y por lo tanto podrían tomar ventajas en el marcador. Por otro lado, si los equipos ganadores terminan de tomar ventaja en el tercer periodo de juego, eso explicaría por qué los perdedores presionan tanto el último periodo (para intentar remontar un marcador ya en desventaja).

Finalmente, cabe destacar que de cada uno de los equipos analizados se observaron tres partidos, de manera que en esos mismos tres partidos el mismo equipo se enfrentaba al mismo rival (playoff al mejor de cinco partidos). Además, en los tres partidos analizados, salvo en un emparejamiento, los tres partidos fueron ganados por el mismo equipo. En este sentido, cabe destacar que dichos condicionantes, pueden estar influenciados en los resultados finales del estudio, además de que se aprecian diferencias importantes en el porcentaje de fases de ataque de cada uno de los equipos, con respecto al total de fases analizadas, lo que puede influir en las medias totales. Estas diferencias de porcentajes, están relacionadas con los propios sistemas y estilos de juego, de manera que unos equipos utilizaban estilos de juego basados en el contraataque (muchas fases de ataque en sus partidos), y otros en el juego estático (pocas fases de ataque). 
Ortega, E.; Fernández, R.; Ubal. M.; Lorenzo, A.; Sampaio, J. (2010). Indicadores de rendimiento defensivo en baloncesto en los ganadores y perdedores. Revista Internacional de Ciencias del Deporte. 19(6), $100-111$. http://www.cafyd.com/REVISTA/01901.pdf

Estos condicionantes suponen que los datos deberán ser analizados con cautela, si bien el propio tamaño de la muestra, así como el importante número de equipos que forman parte de la muestra, aporta valor a los datos obtenidos.

En cualquier caso, cabe indicar que sería muy interesante incrementar el número de fases de ataque analizadas, incluyendo fases de ataque de otros partidos y otros equipos, así como fases de ataque de diferentes temporadas, lo que podría dar una valoración más global y realista del tipo de defensa utilizado en la Liga de Baloncesto Chilena.

\section{Conclusiones}

$>$ A lo largo de todo el partido, los equipos ganadores alternan más entre la defensa individual y zonal. En cambio los perdedores alternan entre individual, zonal y mixta.

$>$ Las diferencias en el marcador se producen en el primer y tercer periodo de juego. En esos momentos los perdedores permiten más puntos.

$>$ Los perdedores presionan más la transición ofensiva en los dos últimos periodos de juego.

$>$ En el cuarto periodo, el porcentaje de rebotes defensivos capturados es mayor en los equipos perdedores.

$>$ Los cambios defensivos, ayudas sobre el hombre con balón y pases interiores permitidos, no diferencian a ganadores de perdedores en ningún periodo.

\section{Referencias Bibliográficas}

Anguera, M.T. (1999). Observación en deporte y conducta Cinésico-Motriz: Aplicaciones. Barcelona: Ediciones de la Universidad de Barcelona.

Anguera, M.T. (2004). Hacia la búsqueda de estructuras regulares en la observación del fútbol: detección de patrones temporales. Cultura, Ciencia y Deporte, 1(1), 15-20.

Cárdenas, D.; Piñar, M.I.; Sánchez, M., y Pintor, D. (1999). Análisis del juego interior en baloncesto. Motricidad, 5, 87-110.

De Rose, D. (2004). Statistical analysis of basketball performance indicators according to home/away games and winning and losing teams Journal of Human Movement Studies, 47(4), 327-366.

Farinha, V. y Tavares, F. (2007). Análise das acções defensivas em equipas de basquetebol sénior masculino. In F. Tavares (Ed.), 10 Congresso Internacional de Jogos Desportivos. Porto:Universidade de Porto Paper presentado en el I Congresso Internacional de Jogos Desportivos, Porto.

Fernández, R.; Ortega, E., \& Ducoing, E. (2007). Relationship between the number of passes made with the points made per phase of offense and with the final result in basketball. Iberian Congress on Basketball Research, 4, 58-60

Forde, K. (2002). Relationship between rebounding margin and winning percentage in the North Central Region. Tesis, Southwest State University, Minnesota. 
Ortega, E.; Fernández, R.; Ubal. M.; Lorenzo, A.; Sampaio, J. (2010). Indicadores de rendimiento defensivo en baloncesto en los ganadores y perdedores. Revista Internacional de Ciencias del Deporte. 19(6), 100-111. http://www.cafyd.com/REVISTA/01901.pdf

García, A.; Parejo, I.; De la Cruz, E.; Domínguez, A. M., \& Saavedra, J. M. (2007). Differences in basketball game statistics between winning and losing teams in the Spanish EBA league. I berian congress on Basketball Research, 4, 76-78

García, J.; Cañadas, M.; Parejo, I.; Feu, S., \& Jiménez, A. C. (2007b). Effect of fatigue on discriminative game-related statistics in an under-20 basketball league. Iberian congress on Basketball Research, 4, 118-121.

Goldstein, S. (2003). La Biblia del Entrenador de Baloncesto. Barcelona: Paidotribo.

Gómez, M. A.; Lorenzo, A.; Ortega, E.; Sampaio, J., y Ibáñez, S.J. (2007). Diferencias en las estadísticas de juego entre bases, aleros y pívots en baloncesto femenino. Cultura, Ciencia y Deporte, 2(6), 139-144.

Gómez, M. A.; Lorenzo, A.; Sampaio, J.; \& Ibáñez, S. J. (2006a). Differences in gamerelated statistics between winning and losing teams in women's basketball Journal of Human Movement Studies, 51(5), 357-369.

Gómez, M. A.; Tsamourtzis, E., \& Lorenzo, A. (2006b). Defensive systems in basketball ball possessions. International J ournal of Performance Analysis in Sport, 6(1), 98-107.

Gómez, M. A. (2007). Estudio de la actividad competitiva en baloncesto masculino y femenino mediante el análisis de las estadísticas de juego y las posesiones de balón. Tesis Doctoral. Madrid: Universidad Politécnica de Madrid.

Hofer, H. (1990). A study of men's college basketball statistics and their relationship to winning and losing. Tesis, Texas: East Texas State University.

Ibáñez, S.J.; Sampaio, J.; Saenz-Lopez, P.; Giménez, J., \& Janeira, M.A. (2003). Game statistics discriminating the final outcome of Junior World Basketball Championship matches. J ournal of Human Movement Studies, 45(1), 1-19.

Ibáñez, S.; García, J.; Cañadas, M.; Moreno, M.; Lorenzo, A., y Gómez, M. (2007a). Estudio de la eficacia del lanzamiento a canasta en la liga E.B.A. In F. Tavares (Ed.), 1o Congresso Internacional de Jogos Desportivos. Porto:Universidade de Porto

Ibáñez, S. J.; Feu, S.; García, J.; Cañadas, M., \& Parejo, I. (2007b). Multifactorial study of shot efficacy in the spanish professional basketball league. Iberian Congress on Basketball Research, 4, 54-57

Iglesias, D.; Cárdenas, D., y Alarcón, F. (2007). La comunicación durante la intervención didáctica del entrenador. Consideraciones para el desarrollo del conocimiento táctico y la mejora en la toma de decisiones en Baloncesto. Cultura, Ciencia y Deporte, 7(3), 43-50.

International Basketball Federation, F. I. B. A. (2000). Reglas Oficiales de Baloncesto 2000. Extraídas en Agosto de 2007 de: http://www.fibaamericas.com/reglas.asp

Kantor, S. (2004). The Role of Offense and Defense in 80/20 Basketball. Coach \& Athletic Director, 74(2), 26-28.

Mexas, K.; Tsitskaris, G.; Kyriakou, D., \& Garefis, A. (2005). Comparison of effectiveness of organized offences between two different championships in high level basketball. International J ournal of Performance Analysis in Sport, 5(1), 72-82.

Montaner, C. y Montaner, A.M. (2004). Estudio comparativo del tiempo de posesión y sus efectos en el juego entre un equipo masculino y uno femenino de baloncesto de élite. RendimientoDeportivo.com, 9. 
Ortega, E.; Fernández, R.; Ubal. M.; Lorenzo, A.; Sampaio, J. (2010). Indicadores de rendimiento defensivo en baloncesto en los ganadores y perdedores. Revista Internacional de Ciencias del Deporte. 19(6), 100-111. http://www.cafyd.com/REVISTA/01901.pdf

Olivera, J. (2003). 1250 Ejercicios y Juegos en Baloncesto (5 ed. Vol. 1). Barcelona: Paidotribo.

Oreste, H. (1985). 115 Principios del Básquetbol. Santiago.

Ortega, E.; Cárdenas, D.; Sainz de Baranda, P., \& Palao, J.M. (2006). Differences between Winning and Losing Teams in Basketball Games in Formation Years (14-16 Years Old). International J ournal of Applied Sport Science, 18(2), 1-11

Ortega, E. (2006). La Competición como Medio Formativo en el Baloncesto. Sevilla: WANCEULEN Editorial Deportiva.

Ortega, E. y Fernández, R. (2007). Differences in 3-point shots between winning and losing teams in formative years of basketball play. Iberian Congress on Basketball Research, 4,33-37.

Ortega, E.; Fernández, R.; Gómez, M. A.; Lorenzo, A.; Ibáñez, S. J., y Sampaio, J. (2007). Análisis de las variables que anteceden al lanzamiento de 3 puntos en baloncesto de alto rendimiento y la repercusión en su eficacia. In F. Tavares (Ed.), 1o Congresso Internacional de Jogos Desportivos. Porto:Universidade de Porto.

Quidel, J. M.; Sepúlveda, A.; Soto, E.; Vargas, J., y Zapata, G. (1999). Propuesta metodológica de enseñanza de los fundamentos básicos defensivos en básquetbol para una etapa inicial de aprendizaje. Tesis, Santiago de Chile: Universidad Metropolitana de Ciencias de la Educación.

Sampaio, J. (1998). Los indicadores estadísticos más determinantes en el resultado final de los partidos de básquetbol. Lectura, educación física y deportes, 11.

Sampaio, J.; Lorenzo, A., y Ribero, C. (2006). Momentos críticos en los partidos de baloncesto: metodología para identificación y análisis de los acontecimientos precedentes, Cultura, Ciencia y Deporte, 5 (2), 83-88.

Sanz, I. y Gutiérrez, P. (2004). El análisis del juego ofensivo en baloncesto: un paso más allá de la estadística convencional. RendimientoDeportivo.com, 7.

Taylor, D. (2007). Performance Efficiency Rating for Basketball. Coach \& Athletic Director, 76(9), 26-27.

Trninic, S.; Milanovic, D.; Blaskovic, M.; Birkic, Z., \& Dizdar, D. (1995). The influence of defensive and offensive rebounds on the final score in a basketball game. Kineziologija, 27(2), 44-49.

Wissel, H. (2000). Baloncesto. Aprender y Progresar (3 ed.). Barcelona: Paidotribo. 